\title{
Draft Genome Sequence of a Virulent Strain of Pasteurella Multocida Isolated From Alpaca
}

Raquel Enma Hurtado1, Flavia Aburjaile², Diego Mariano², Marcus Vinicius Canário², Leandro Benevides², Daniel Antonio Fernandez ${ }^{1}$, Nataly Olivia Allasi ${ }^{1}$, Rocio Rimac ${ }^{1}$, Julio Eduardo Juscamayta ${ }^{1}$, Jorge Enrique

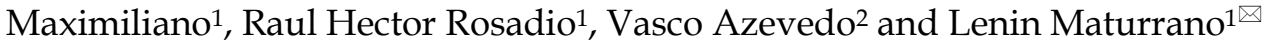

1. Laboratory of Molecular Biology and Genetics, Veterinary Medicine Faculty, San Marcos University, Lima, Peru;

2. Laboratory of Cellular and Molecular Genetics, Federal University of Minas Gerais, Belo Horizonte, Minas Gerais, Brazil.

$\triangle$ Corresponding author: Tel.: +551 956533581. E-mail: amaturranoh@unmsm.edu.pe (L. Maturrano)

(C) Ivyspring International Publisher. This is an open access article distributed under the terms of the Creative Commons Attribution (CC BY-NC) license (https://creativecommons.org/licenses/by-nc/4.0/). See http://ivyspring.com/terms for full terms and conditions.

Received: 2017.01.22; Accepted: 2017.05.14; Published: 2017.06.28

\begin{abstract}
Pasteurella multocida is one of the most frequently isolated bacteria in acute pneumonia cases, being responsible for high mortality rates in Peruvian young alpacas, with consequent social and economic costs. Here we report the genome sequence of $P$. multocida strain UNMSM, isolated from the lung of an alpaca diagnosed with pneumonia, in Peru. The genome consists of 2,439,814 base pairs assembled into 82 contigs and 2,252 protein encoding genes, revealing the presence of known virulence-associated genes (ompH, ompA, tonB, tbpA, nanA, nanB, nanH, sodA, sodC, plpB and toxA). Further analysis could provide insights about bacterial pathogenesis and control strategies of this disease in Peruvian alpacas.
\end{abstract}

Key words: Alpaca, genome, pasteurellosis, pneumonia.

\section{Introduction}

Pasteurella multocida is a commensal bacteria from the upper respiratory tract [1], which affects a wide range of hosts $[2,3]$. This bacteria is the primary agent of many infections such as; avian cholera, hemorrhagic septicemia in ungulates, atrophic rhinitis in pigs and snuffles in rabbits [3], and acts as a secondary agent in infectious pneumonia, including cases of acute or chronic pneumonia in different hosts such as swine, calves, sheep, bovine and alpaca [3-5].

In Peru, alpaca raising represents an important economic activity for the High Andean population. However, acute pneumonia causes high mortality rates in young alpacas, in which $P$. multocida has been principally isolated [6]. P. multocida has a large number of virulence factors that play a role in pathogenesis, including capsule, lipopolysaccharide, fimbriae, adhesins, toxins, outer membrane proteins, iron regulated and iron acquisition proteins, acquisition proteins, hyaluronidase and sialidase [6].
In this study, we announce the draft genome of $P$. multocida strain UNMSM isolated from an alpaca lung affected with pneumonia.

Pasteurella multocida strain UNMSM is a gram negative, short rod shaped bacteria, oxidase and catalase positive and nonhemolytic, with approximate measures of $0.3-0.6 \mu \mathrm{m}$ in width and $0.8-2.0 \mu \mathrm{m}$ in length (Figure 1). Genome sequencing was performed using Illumina Hiseq sequencing platform. The paired-end library contained inserts of an average size of $100 \mathrm{bp}$. De novo assembly was performed using Edena v3.131028 and SIMBA v1.4 software [7], which produced 82 contigs, with a N50 value of $70,838,2.4 \mathrm{Mb}$ of size and mean depth coverage $\sim 400$-fold. The genome was annotated using the Rapid Annotations using Subsystems Technology (RAST) [8], following by manual curation of the predicted CDSs (Coding Sequences). The genome presents GC content around $40.2 \%$. A total of 2,434 
genes were predicted, of which 2,252 were protein-coding genes, eight rRNA genes (four $16 S$ rRNA, one 23S rRNA and three 5S rRNA genes) and 55 were tRNA genes. In table 1, a few summary statistics of the genome are presented.

Table 1. Genome statistics of $P$. multocida strain UNMSM.

\begin{tabular}{lll}
\hline Attribute & Value & $\%$ of Total \\
\hline Genome size (bp) & $2,439,814$ & $100.00 \%$ \\
DNA coding region(bp) & $2,066,315$ & $84.69 \%$ \\
DNA G+C content (bp) & 979,694 & $40.15 \%$ \\
Total genes & 2,434 & $100.00 \%$ \\
Pseudogenes & 115 & $4.72 \%$ \\
Genes assigned to COGs & 1,921 & $78.92 \%$ \\
Genes with Pfam domains & 1,183 & $48.60 \%$ \\
Genes with signal peptides & 173 & $7.10 \%$ \\
Genes with transmembrane helices & 490 & $20.13 \%$ \\
CRISPR repeats & 1 & - \\
\hline
\end{tabular}

* The total is based on either the size of the genome in base pairs or the total number of genes in the annotated genome.

The Cluster of Orthologous Genes (COG) [8] assignments were done using CGView Comparison Tool (CCT), and the presence of protein domains was predicted by Pfam. The signal peptides were identified with the SignalP 4.0 software [9], transmembrane helices were classified by method of Krogh and collaborators method [10], and the CRISPR motif was detected with a web tool described by Grissa and collaborators [11] (Table 1). The functional analysis obtained from Rapid Annotation using Subsystem Technology (RAST) revealed 395 collections of functionally related protein families (Figure 2). The genome has 54 genes responsible for iron acquisition and metabolism; 42 genes involved in virulence, disease and defense; 74 genes involved in membrane transport, and 75 genes related to phages, prophages and transposable elements. Analysis of genes showed that this strain belongs to capsular type $\mathrm{A}$, and the presence virulence associated genes, including outer membrane proteins as ompH (UR07_03150), and ompA (UR07_05380); hyaluronidases as nanA (UR07_06210), nanB (UR07_07035) and nanH (UR07_07095); iron acquisition related genes, ton $B$ (UR07_07610) and tbp A (UR07_03090); genes involved in sialic acid metabolism as sodA (UR07_00380) and sodC (UR07_00680); a lipoprotein, plpB (UR07_09985) and toxA (UR07_03905), a dermonecrotic toxin [5-12].

This work provides a better understanding of $P$. multocida UNMSM, the first genome isolated from alpaca. Future studies in vitro and in vivo associated with virulence genes predicted in P. multocida, will improve understanding of their pathogenicity and provide an assessment of new targets for the use of vaccines and drugs, which may be important for the control of pneumonia case numbers in alpacas. The genome projects of $P$. multocida strain UNMSM have been deposited in GenBank under the following accession numbers, LGRE00000000.

\section{Acknowledgements}

This work was conducted by Laboratory of Cellular and Molecular Genetics in Federal University of Minas Gerais and Veterinary Medicine Faculty in National University of San Marcos and present work was financially supported by the Programa Nacional de Innovación para la Competitividad y Productividad (Innóvate Perú), under the contract 133-FINCyT-IB-2013, as part of the project "Reverse Vaccinology: Developing a new generation vaccine for the control and/or prevention pasteurellosis pneumonia in alpacas".
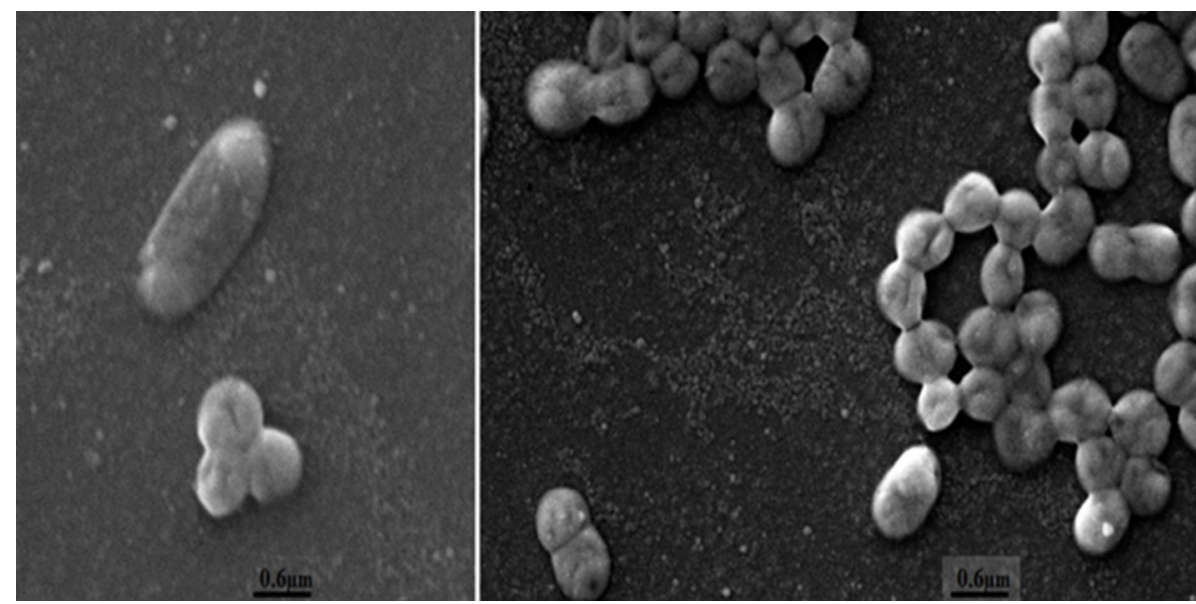

Figure 1. Scanning electron micrograph of cells of $P$. multocida strain UNMSM. The scale bar represents $0.6 \mu \mathrm{m}$. 


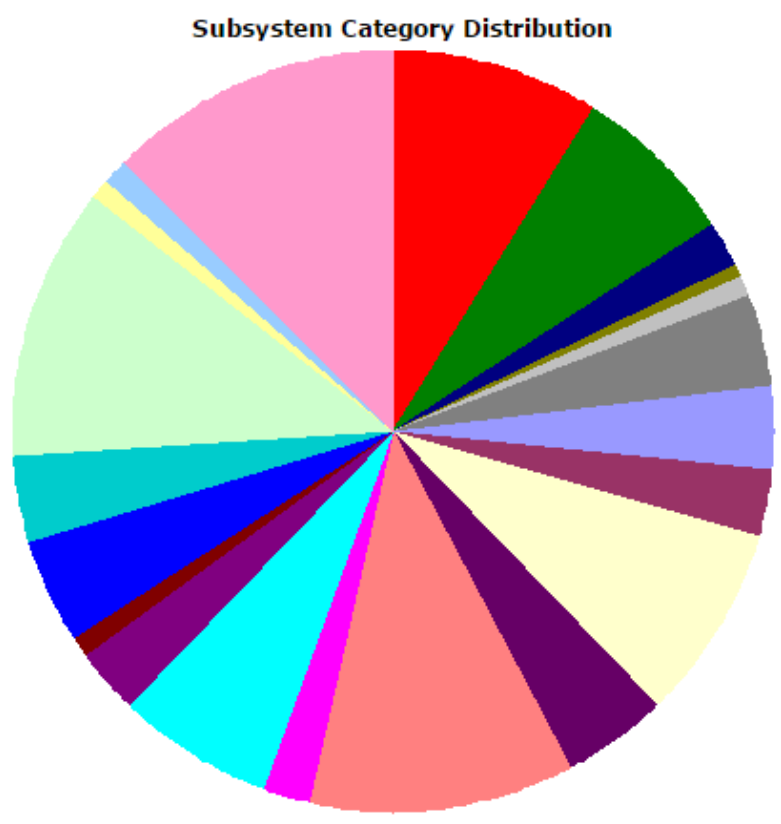

Subsystem Feature Counts

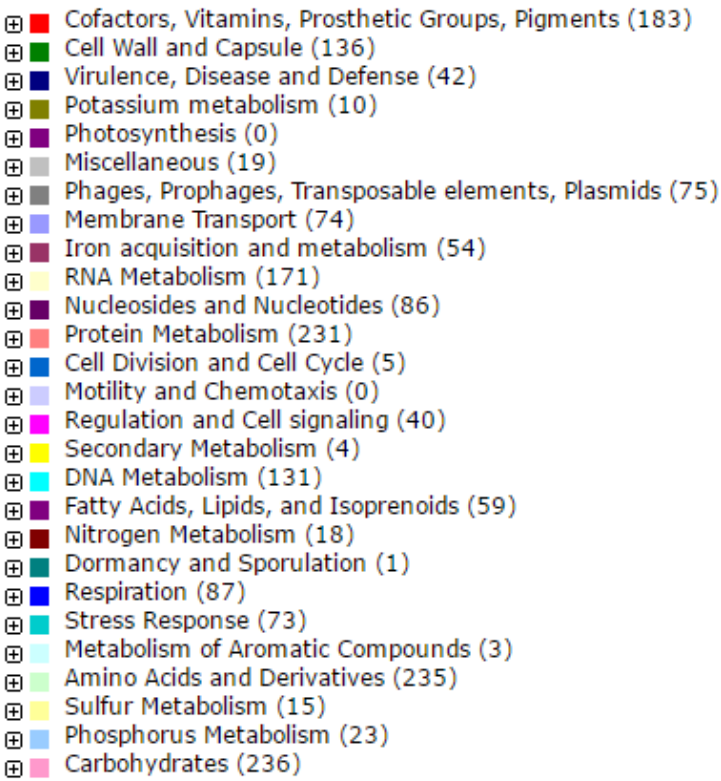

Figure 2. The overview of subsystem category coverage P. multocida UNMSM genome based on RAST server.

\section{Competing Interests}

The authors have declared that no competing interest exists.

\section{References}

1. Timoney JF. Guillespie JA, Scott FW, Barlough JE. The genus Pasteurella. In. Hagan and Bruner's Microbiology and Infectious Diseases of Domestic Animals, 8th ed. Ithaca: Cornell University Press; 1989: 104-116.

2. Christensen H, Bisgaard M. The genus Pasteurella. In: Dworkin M, Falkow S, Rosenberg E, Schleifer KH, Stackebrandt E, eds. The Prokaryotes, 3rd ed. Berlin: Springer New York; 2006: 1062-1090.

3. Wilkie IW, Harper M, Boyce JD, Adler B. Pasteurella multocida: Diseases and Pathogenesis. In: Aktories K, Orth JHC, Adler B, eds. Pasteurella Multocida. Berlin, Heidelberg: Springer Berlin Heidelberg; 2012: 1-22.

4. Rosadio R, Cirilo E, Manchego A, Rivera H. Respiratory syncytial and parainfluenza type 3 viruses coexisting with Pasteurella multocida and Mannheimia hemolytic in acute pneumonia of neonatal alpacas. Small Rumin. Res. 2011; 97: 110-6.
5. Ameghino E, De Martíni J. Mortalidad de crías de alpacas. Bol. Divulg. Inst. Vet. Investig. Trop. Altura IVITA UNMSM. 1991;71-80.

6. Ramírez A. Enfermedades infecciosas en camélidos sudamericanos. Lima, Perú: XII Reunión científica anual APPA; 1989.

7. Hernandez D, Francois P, Farinelli L, Osteras M, Schrenzel J. De novo bacterial genome sequencing: Millions of very short reads assembled on a desktop computer. Genome Res. 2008; 18: 802-9.

8. Grant JR, Arantes AS, Stothard P. Comparing thousands of circular genomes using the CGView Comparison Tool. BMC Genomics. 2012;13: 202.

9. Petersen TN, Brunak S, von Heijne G, Nielsen H. SignalP 4.0: discriminating signal peptides from transmembrane regions. Nat. Methods. 2011; 8: 785-6.

10. Krogh A, Larsson B, von Heijne G, Sonnhammer EL. Predicting transmembrane protein topology with a hidden Markov model: application to complete genomes. J. Mol. Biol. 2001; 305: 567-80.

11. Grissa I, Vergnaud G, Pourcel C. CRISPRFinder: a web tool to identify clustered regularly interspaced short palindromic repeats. Nucleic Acids Res. 2007; 35: W52-7.

12. Katsuda K, Hoshinoo K, Ueno Y, Kohmoto M, Mikami O. Virulence genes and antimicrobial susceptibility in Pasteurella multocida isolates from calves. Vet. Microbiol. 2013; 167: 737-41. 